\title{
Matching Security Policies to Application Needs
}

\author{
Claudia Eckert \\ Technical University of Munich, \\ Institut für Informatik, 80290 München, Germany \\ e-mail : eckertc@informatik.tu-muenchen.de
}

\begin{abstract}
The issue of developing complex secure systems is still a great challenge. We claim that in contrast to the well known bottom-up oriented approaches secure concurrent systems should be developed top-down starting with a formal top-level specification. A framework for developing secure systems is needed, which offers means to specify security requirements adapted to the specific demands of application areas. In addition, an appropriate security model is needed to formally describe the behavior and the security properties of systems. We will present a uniform framework which is appropriate to match security policies to application needs.

Secure concurrent systems are modeled with two different levels of abstraction. The action model provides a sound and fine-grained basis to formalize security properties of the system. In order to ease system modeling we introduce the object security model by systematically coarsening the action model. In addition to our security model we will present a security requirement logic. Security policies tailored to the specific requirements of applications may by specified with the formulas of the logic. The security requirement logic allows to specify different security policies such as access control and information flow policies in a uniform way, and allows to compare these policies.

Key words : formal model, security model, security policy, access control, information flow control
\end{abstract}

\section{Introduction}

The issue of developing complex secure systems is still a great challenge. Ad hoc solutions which extend existing operating system services with additional security mechanisms and services to enforce security requirements are error prone and not satisfactory. We claim that in contrast to the well known bottom-up oriented approaches secure concurrent systems should be developed top-down starting with a formal top-level specification which contains the integrated specification of security properties. According to the top-down approach the systems are realized on several levels of abstraction by stepwise refining and implementing the specified properties. An integrated approach enables to verify the enforcement of the properties on each level of abstraction. The paper will present a formal and uniform framework which offers means to define formal top-level specifications and, in addition, which offers a security model for secure concurrent systems. Within the paper 
we will restrict our attention to the modeling of concurrent secure systems neglecting the efforts needed to realize the modeled systems in a physically distributed environment. The security requirements of a system specify the allowed or disallowed information flows between users, as well as, access restrictions for single users. Within our framework a security requirement logic has been developed which enables to specify security requirements tailored to application needs. The security requirement logic will be presented.

To describe the behavior and the security properties of a concurrent system an appropriate security model is needed. The lesson learned from studying the well known models is that a security model for concurrent systems is required which enables to model access properties, as well as, information flow properties adapted to the needs of individual application systems. The model should be easy to understand and to use, nevertheless, it must be sound. To achieve these requirements we propose an object-based approach to design secure systems. A concurrent system can be viewed as a set of active and passive objects interacting by means of method calls. The objects are the protected entities which can be specified with fine-grained granularity. Objects are only accessible via well-formed operations comparable with well-formed transactions in the Clark-Wilson model (c.f. [CW87]). An object-based setting provides an appropriate basis to determine fine-grained access rights. The active objects are the subjects of the system acting in behalf of human users. From the point of view of a user the object abstraction is an appropriate level of abstraction to determine access restrictions for users concerning protected objects, and to determine information flow restrictions between users. In order to formalize the behavior of the system we introduce the action model. An action model provides the formal basis needed to define the security properties of a system.

Based on the action model a logic for specifying security requirements will be introduced. Security requirements are expressed by means of logic formulas. The formulas of the logic offer a powerful formalism to specify access control policies, as well as, information flow policies. Using the logic we can specify well known security policies in a uniform manner, for instance, the multi-level security policy [BL75], or the Chinese Wall policy [BN89], as well as, application specific security policies. The model used in our approach may be characterized as information flow model with special support for data integrity based on a computation model supporting concurrency.

The rest of the paper will be organized as follows. In section 2 we introduce the action model and the object security model for secure concurrent systems and define basic security properties. The logic to specify security requirements is defined in section 3 . Some examples, for instance the specification of the multi-level security policy and of the Chinese Wall policy, demonstrate the expressiveness of the logic. Finally, we will introduce the ordering on policies which enables us to systematically compare security policies. Section 4 concludes the paper. 


\section{Models}

In this section two levels of abstraction to model secure concurrent systems namely the action model and the object security model are presented. Within the framework of the models basic security properties can be formalized.

\subsection{Action Model}

A concurrent system is modeled as an abstract machine by an action model given in definition 1.

Definition 1 Action Model:

A concurrent system is modeled by a tuple $\mathcal{A M}=\left(V, \Sigma, \mathcal{A}, \Gamma, S_{0}\right)$ with:

1. $V$ is the set of state objects.

For each object $v \in V$ the set of values of object $v$ is defined by Range $(v)$.

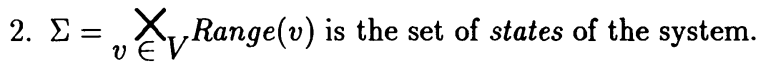

Given a state $s \in \Sigma, s[v] \in$ Range $(v)$ describes the value of object $v \in V$ in state $s$.

3. $\mathcal{A}$ is the set of atomic actions. For each $a \in \mathcal{A}, E N_{a}$ describes the enabling condition of $a, D_{a} \subseteq V$ is the domain, and $R_{a} \subseteq V$ is the range of $a$.

A sequence of actions is denoted by $\langle A\rangle$.

4. $\Gamma$ is the state transition relation with $\Gamma \subseteq \Sigma \times \mathcal{A} \times \Sigma$.

An action $a \in \mathcal{A}$ is called enabled in a state $s \in \Sigma: \Longleftrightarrow$ the enabling condition $E N_{a}$ of $a$ is true in state $s$.

The mapping ${ }^{1}$ en $: \Sigma \longrightarrow \mathcal{P}(\mathcal{A})$ describes the set of enabled actions in a state $s$. A state transition from $s$ to $s^{\prime}$ by executing action $a$ is denoted by $s \stackrel{a}{\longrightarrow} s^{\prime}$.

5. $S_{0} \subseteq \Sigma$ is the set of initial states.

The abstract machine modeled by an action model consists of a set of state objects $V$, for example variables, a set of atomic actions $\mathcal{A}$ defining the computational steps of the machine, the set of states $\Sigma$ defined by the set of values of the state objects, a state transition relation $\Gamma$ defining the state transitions of the machine and a set of initial states $S_{0}$. The execution of an action causes a state change. Each action $a$ may contain a precondition, the so called enabling condition $E N_{a}$, which must be true before the action may be executed. In addition, for each action two sets of state objects, the domain set $D_{a}$

\footnotetext{
${ }^{1} \mathcal{P}$ denotes the power set.
} 
and the range set $R_{a}$ ared defined. The state objects of the domain set may be read and the objects of the range set may be modified during the execution of the action. Starting with an initial state $s_{0} \in S_{0}$ the system evolves by executing enabled atomic actions. The state transition relation selects non deterministically an executable action and performs the state change according to the defined effects of the action.

A sequence of actions is called accepted in $\mathcal{A M}$ if the first action of the sequence is enabled in one of the initial states of $\mathcal{A M}$. Furthermore, each action of the sequence must be enabled in the state produced by the execution of its predecessor action within the sequence.

The sequence of states associated with an accepted sequence $\langle A\rangle$ of actions is called a computation $\sigma_{A}$ of the system.

\section{Notation:}

Let $\mathcal{A}^{\Delta}$ denote the set of accepted sequences of actions and let $\Sigma^{\Delta}$ denote the set of computations of a system.

Let $b \in\langle A\rangle$ denote the membership of action $b$ in the action sequence $\langle A\rangle$.

The granularity and formal semantics of actions depend on the selected level of abstraction. Actions may represent, for instance, atomic statements of a high-level programming language or processor instructions at a lower level of abstraction. In spite of defining the formal semantics of a specific set of actions we will introduce an abstract notion of the effect of an action. This level of abstraction is sufficient to formally define the security properties we are interested in. Moreover, it offers the ability to select the appropriate level of abstraction due to modeling requirements.

Definition 2 Effect of an action:

Given a system $\mathcal{A M}$. The effect of an action $b \in \mathcal{A}$ in a computation $\sigma_{A}$ is defined by the function $e f_{a}$.

$$
\begin{gathered}
e f_{a}: \Sigma^{\Delta} \times \mathcal{A} \longrightarrow \mathcal{P}\left(\Sigma \times V \times \bigcup_{v \in V} \operatorname{Range}(v)\right) \cup\{\text { nil }\} \quad \text { with } \\
e f_{a}\left(\sigma_{A}, b\right) \triangleq \begin{cases}\left\{\left(s^{\prime}, v, s^{\prime}[v]\right) \mid v \in V: s, s^{\prime} \in \sigma_{A} \wedge s \stackrel{b}{\longrightarrow} s^{\prime} \text { and } s[v] \neq s^{\prime}[v]\right\} & \text { if } b \in<A> \\
\{n i l\} & \text { otherwise }\end{cases}
\end{gathered}
$$

The effect of an action $b$ executed within a computation $\sigma_{A}$ is given by a set of triples. Each triple stores a modified state object $v$, the state $s^{\prime}$ after the modification and the new value $s^{\prime}[v]$ of object $v$.

Now we are able to define the security properties of a system $\mathcal{A M}$ with respect to information flows between actions. The security properties describe the relationships between actions according to the abilities of an action to change the effects of other actions, i.e. to influence their effects, and to observe the effects of other actions. 


\subsubsection{Influence and Observation}

We say that an action $a$ influences the effects of an action $b$ by means of a member $v$ of the domain $D_{b}$ of $b$ if there exists an information flow from action $a$ to action $b$ through object $v$ and if this information flow may be passed on to other actions by executing $b$. The formal definition is given in definition 4. For shortening the definition we first introduce a function mask which formalizes the result of purging the effects of an action from a given action sequence.

Definition 3 Masking effects:

Given a system $\mathcal{A M}$ and a special action $a_{i d}$ with $e f_{a}\left(\sigma_{A}, a_{i d}\right)=\emptyset$ for each computation $\sigma_{A}$ of the system.

The function mask is defined by: mask $: \mathcal{A}^{\Delta} \times \mathcal{A} \longrightarrow \mathcal{A}^{\Delta} \cup\{$ nil $\}$ with

$$
\operatorname{mask}(<A>, a) \triangleq\left\{\begin{array}{lll}
\langle\bar{A}>\text { if } & <A>=<a_{1}, \ldots, a_{k}>\text { and } \\
\text { nil }\} & \text { otherwise, } & \exists a_{j} \in<>\text { with } a_{j}=a
\end{array}\right.
$$

$<\bar{A}>$ is defined by: $\langle\bar{A}>\triangleq<f(1), \ldots, f(k)>$ with

$f(i) \triangleq\left\{\begin{array}{lll}a_{i} \quad(\text { if } i<j) \text { or }(\text { if } & (i>j) \text { and } \\ & \left.\left(a_{i} \in \text { en }\left(\overline{s_{i-1}}\right) \text { with } \sigma_{f(1), \ldots, f(i-1)}=\overline{s_{0}}, \overline{s_{1}}, \ldots, \overline{s_{i-1}}\right)\right) \\ a_{i d} & \text { otherwise }\end{array}\right.$

Given an accepted sequence of actions $\langle A\rangle$ and an action $a \in<A>$ the function mask generates the sequence of actions $\langle\bar{A}\rangle$ with the following four properties. (1) $<\bar{A}\rangle$ contains all actions of $\langle A\rangle$ which have been executed prior to $a$. (2) $\langle\bar{A}\rangle$ contains the effectless action $a_{i d}$ substituted for $a$. (3) $<\bar{A}>$ contains all actions which are still enabled in the state sequence associated with $\langle\bar{A}\rangle$, and $(4)\langle\bar{A}\rangle$ contains the effectless action $a_{i d}$ substituted for each action $b$ of $\langle A\rangle$ which is unabled in computation $\sigma_{\bar{A}}$ associated with $\langle\bar{A}\rangle$.

\section{Notation :}

Given two computations $\sigma, \sigma^{\prime} \in \Sigma^{\Delta}$ with $\sigma=s_{0}, \ldots, s_{j}$ and $\sigma^{\prime}=s_{0}, \ldots, s_{r}$ and the state object $v \in V$.

$$
\tilde{s_{r}} \triangleq s_{r}\left[\begin{array}{l}
v \\
s_{j}[v]
\end{array}\right]
$$

denotes the substitution of the value of object $v$ in state $s_{r} \in \sigma^{\prime}$ by the value of $v$ in state $s_{j} \in \sigma$. That is, in state $\tilde{s}_{r}$ all state objects $x \neq v$ contain the value of state $s_{r}$, that is $\tilde{s_{r}}[x]=s_{r}[x]$, and for $v$ holds: $\tilde{s_{r}}[v]=s_{j}[v]$.

Note: The evaluation of predicates in a state of a computation is defined by the function $\omega$. 
Definition 4 Influences:

Given a system $\mathcal{A M}$, the finite prefix $\left\langle A>\right.$ of an accepted sequence $\left\langle A^{\prime}\right\rangle \in \mathcal{A}^{\Delta}$, $s_{0} \stackrel{\langle A>}{\longrightarrow} s_{k}$ and $s_{0} \in S_{0}$ with the associated computation $\sigma_{A}$, two actions $a, b \in \mathcal{A}$, an object $v \in V$, and a state $s_{l} \in \sigma_{A}$.

The action $a$ directly influences the action $b$ in the sequence $s_{l}, \ldots, s_{k}$ of $\sigma_{A}$ by means of the state object $v$ if and only if the predicate $i n f_{a}^{d}(l, a, b, v)$ is true in state $s_{k}$.

(C1) $\exists s_{i}, s_{j} \in \sigma_{A}$ with $i, j<k \wedge i<j \wedge j \geq l \wedge$

$\exists s_{i}^{\prime}, s_{j}^{\prime} \in \sigma_{A}$ with $i^{\prime}, j^{\prime} \leq k$ with $i^{\prime} \leq j$ and

$s_{i} \stackrel{a}{\longrightarrow} s_{i^{\prime}} \wedge s_{j} \stackrel{b}{\longrightarrow} s_{j^{\prime}}$ and $v \in D_{b}$ and

$<\bar{A}>\triangleq \operatorname{mask}(<A>, a)$ with $\sigma_{\bar{A}}=\overline{s_{0}}, \overline{s_{1}}, \ldots, \overline{s_{k}}$.

and

(C2) $b \notin e n\left(\overline{s_{j}}\right)$

or

(C1) $\exists s_{i}, s_{j} \in \sigma_{A}$ with $i, j<k \wedge i<j \wedge j \geq l \wedge$

$\exists s_{i}^{\prime}, s_{j}^{\prime} \in \sigma_{A}$ with $i^{\prime}, j^{\prime} \leq k$ with $i^{\prime} \leq j$ and

$s_{i} \stackrel{a}{\longrightarrow} s_{i^{\prime}} \wedge s_{j} \stackrel{b}{\longrightarrow} s_{j^{\prime}}$ and $v \in D_{b}$ and

$<\bar{A}>\triangleq \operatorname{mask}(<A>, a)$ with $\sigma_{\bar{A}}=\overline{s_{0}}, \overline{s_{1}}, \ldots, \overline{s_{k}}$.

and

(C3) $b \in e n\left(\overline{s_{j}}\right) \wedge s_{j}[v] \neq \overline{s_{j}}[v] \wedge \exists x \in R_{b}: s_{j^{\prime}}[x] \neq \overline{s_{j^{\prime}}}[x]$.

and

$(C 4)$

$\tilde{s_{j}} \triangleq s_{j}\left[\frac{v}{s_{j}}[v]\right]$ and $b \notin e n\left(\tilde{s_{j}}\right)$

or $b \in \operatorname{en}\left(\tilde{s_{j}}\right)$ and $\tilde{s_{j}} \stackrel{b}{\longrightarrow} \tilde{s_{j^{\prime}}} \wedge \exists y \in R_{b}: s_{j^{\prime}}[y] \neq \tilde{s_{j^{\prime}}}[y]$.

Condition $C 1$ holds if: Actions $a$ and $b$ have been executed prior to state $s_{k}$. Action $a$ has been executed in a state $s_{i}$ prior to the execution of $b$ in state $s_{j} . b$ has been executed within the sequence $s_{l}, \ldots, s_{k}$, and object $v$ is a member of the domain of $b$, i.e. $v$ may be read during the execution of $b$.

Condition $C 2$ holds if: The action $b$ is unabled in the modified action sequence $\langle\bar{A}\rangle$ masking the effect of action $a$.

If conditions $C 1$ and $C 2$ hold action $a$ directly influences action $b$, because the enabling condition of $b$ depends on the execution of action $a$.

Condition $C 3$ holds if: The action $b$ is still enabled in the modified sequence and the value of $v$ depends on the execution of action $a$, i.e. the object $v$ contains different values in the state $s_{j}$ and $\overline{s_{j}}$ just before the execution of $b$ in $\sigma_{A}$ and in $\sigma_{\bar{A}}$. Moreover, there exists an object $x$ in the range of $b$ which contains different values after the execution of $b$ in the computation $\sigma_{A}$ and in $\sigma_{\bar{A}}$.

Condition $C 4$ holds if: The different effects of $b$ in the computation $\sigma_{A}$ and $\sigma_{\bar{A}}$ are caused 
by the different values of the object $v$ in the domain of $b$ in computation $\sigma_{A}$ and $\sigma_{\bar{A}}$, respectively.

If conditions $C 1, C 3$ and $C 4$ hold action $a$ directly influences action $b$, because the value of the object $x$ in the range of $b$ depends on the execution of action $a$.

Note that definition 4 makes no assumptions about the properties of actions $c$ lying between action $a$ and $b$ in the sequence $\langle A\rangle$. These properties are covered implicitly by the definition above. Consider, for instance, an action $c$ which modifies the value of object $v$ as well and which is unabled in $\langle\bar{A}\rangle$. That is, $v$ contains different values after the execution of $c$ in $\sigma_{A}$ and after the execution of the substitute action $a_{i d}$ for $c$ in $\sigma_{\bar{A}}$. As the masking of $a$ in $\sigma_{\bar{A}}$ causes $c$ to become unabled, action $a$ is responsible for the different values of $v$ and, therefore, influences action $b$. This relationship between actions $a, b$ and $c$ is covered by definition 4 .

In addition to the influence-property of an action we will define the property of an action to observe an influence by means of objects in its range. That is, we will define the property of an action $b$ to observe information flows from other actions by observing the values of objects in its own range. In contrast to the influence-property the observationproperty requires that the influenced action is still enabled after purging the influencing action from the action sequence. That is, the influence on the action is really mirrored by the the value of at least one object in the range of the action.

Definition 5 Observations:

Given the assumptions of definition 4. The action $b$ directly observes the action $a$ in the sequence $s_{l}, \ldots, s_{k}$ of $\sigma_{A}$ by means of the state object $x$ if and only if the predicate $o b s_{a}^{d}(l, b, a, x)$ is true in state $s_{k}$.

$$
\omega\left(o b s_{a}^{d}(l, b, a, x), s_{k}\right)=\text { true }: \Longleftrightarrow
$$

(C1) $\exists v \in D_{b}: \omega\left(i n f_{a}^{d}(l, a, b, v), s_{k}\right)=$ true and $s_{j} \stackrel{b}{\longrightarrow} s_{j^{\prime}}$ and $<\bar{A}\rangle \triangleq \operatorname{mask}(<A>, a)$ with $\sigma_{\bar{A}}=\overline{s_{0}}, \overline{s_{1}}, \ldots, \overline{s_{k}}$

and

$$
\begin{aligned}
& x \in R_{b} \wedge s_{j^{\prime}}[x] \neq \overline{s_{j^{\prime}}}[x] \text { and with } \\
& \tilde{s_{j}} \triangleq s_{j}\left[\begin{array}{l}
v \\
\overline{s_{j}}[v]
\end{array}\right] \text { holds }: \\
& b \in \operatorname{en}\left(\tilde{s_{j}}\right) \wedge s_{j^{\prime}}[x] \neq \tilde{s_{j^{\prime}}}[x] .
\end{aligned}
$$

Condition $C 1$ holds if: Action $b$ is influenced by the execution of action $a$ by means of a state object $v$ in the domain of $b$.

Condition $C 2$ holds if: This influence is mirrored by the value of object $x$ in the range of $b$. That is, there exists an information flow from action $a$ to action $b$ through object $v$ to object $x$. To catch this relationship between $v$ and $x$ we mask the effects of action 
$a$ and substitute the value of $v$ prior to the execution of $b$ with the value of $v$ in the modified action sequence. The different values of $v$ (according to condition $C 1$ ) cause different effects of $b$ observable in different values of object $x$ in computation $\sigma_{A}$ and $\sigma_{\bar{A}}$, respectively.

The properties defined in 4 and 5 can be extended to general definitions of influences and observations between two actions by defining an influence-chain and observationchain, respectively, between two actions $a$ and $b$. The predicate in $f_{a}(l, a, b, v)$ holds if an influence occurs between action $a$ and action $b$ and predicate $o b s_{a}(l, b, a, v)$ holds if action $b$ observes effects of action $a$. The formal definitions are omitted here.

\subsection{Object Security Model}

An action model completely describes the behavior of a system but its fine-grained level of description is cumbersome to handle. So we coarsen the model. This leads to the object security model of a system. Based on the detailed action model the defined influence and observation properties can easily be transmitted to the coarser model. That is, we will define the property of a user to influence the effects of activities performed by other users or to observe the effects of activities performed by other users.

The object security model is given in definition 6 .

Definition 6 Object security model :

The tuple $\mathcal{M S}=\left(\mathcal{A M},(\mathcal{K}, \mathcal{O}, \mathcal{B}, u r e p), R, R L, r l_{b}, r l_{k}\right)$ models a secure concurrent system with

1. $\mathcal{A M}$ is an action model of the system.

2. $(\mathcal{K}, \mathcal{O}, \mathcal{B}$, u rep $)$ is the object model of the system with:

$\mathcal{K}$ is a set of objects and $\mathcal{K}^{\prime} \subseteq \mathcal{K}$ is a set of user representatives.

For each $k \in \mathcal{K}, E(k)$ defines the set of methods of object $k$.

$\mathcal{O}$ is the set of operations. For each $o p \in \mathcal{O}$, in $(o p)$ and out(op) define the set of input and output parameters of operation $o p$.

For each invocation inst_op of an operation op $\in \mathcal{O}$ the mapping $\rho$ defines the sequence of actions $\rho($ inst_op $)=\left\langle a_{1}, \ldots, a_{m}\right\rangle, a_{i} \in \mathcal{A}$, which describes the computational steps of inst_op. $\rho$ (inst_op) is called the action refinement of inst_op.

The set $V$ of state objects contains special objects called access control lists:

$\forall k \in \mathcal{K} \quad \exists a c l_{k} \in V: \forall s \in \Sigma:$

$s\left[a c l_{k}\right]=\left\{\left(k^{\prime}, O P\right) \mid k^{\prime} \in \mathcal{B} \cup R \cup \mathcal{O} \cup \mathcal{K} \wedge O P \subseteq E(k)\right\}$.

$a c l_{k}$ is called the access control list of $k$.

$\mathcal{B}$ is the set of users.

The mapping urep : $\mathcal{B} \longrightarrow \mathcal{P}\left(\mathcal{K}^{\prime}\right)$ associates with each user of the system a set of user representatives: $\forall b, b^{\prime} \in \mathcal{B}: b \neq b^{\prime} \Longrightarrow u \_r e p(b) \cap u \_r e p\left(b^{\prime}\right)=\emptyset$.

The mapping ass_u(ur) : $\mathcal{K}^{\prime} \longrightarrow \mathcal{B}$ associates a user with a user representative: $a s s \_u(u r)=b \Longleftrightarrow u r \in u \_r e p(b)$. 
3. $R$ is a set of access rights ${ }^{2}$, with $\mathcal{O} \subseteq R$.

4. $R L=\left\{R_{1}, \ldots, R_{m}\right\}$ is the set of roles.

5. The mapping $r l_{b}: \mathcal{B} \longrightarrow \mathcal{P}(R L)$ associates with each user a set of roles.

6. The mapping $r l_{k}: \mathcal{K}^{\prime} \longrightarrow R L$ with

$$
r l_{k}(u r)=R_{j}: \Longleftrightarrow \quad \exists b \in \mathcal{B}: R_{j} \in r l_{b}(b) \wedge b=a s s_{-} u(u r)
$$

associates with each user representative $u r$ a role $R_{j}$. A representative $u r$ is active in behalf of user $b=a s s_{-} u(u r)$ in the associated role $r l_{k}(u r)$.

It holds: $\forall b \in \mathcal{B}: \forall u r, u r^{\prime} \in u \_e p(b): u r \neq u r^{\prime} \Longleftrightarrow r l_{k}(u r) \neq r l_{k}\left(u r^{\prime}\right)$.

A concurrent system is modeled by a set of objects $\mathcal{K}$. For each object $k \in \mathcal{K}$ a set of operations or methods $E(k)$ may be defined. The elements of $E(k)$ may be invoked by other objects. The set of all operations of the system is given by $\mathcal{O}$. An object may be active (process) or passive (monitor, module). The set of users is given by the set $\mathcal{B}$. Within the object model each user $b$ is represented by a set of active objects $u r e p(b)$, the so called user representatives, which execute operations in behalf of the user.

Operations are invoked by users and executed by the associated user representatives. Each invocation creates an instance of the operation and the computational steps of the instance are defined by a sequence of actions called action refinement of the operation invocation. An action refinement of an operation invocation starts with an action creating an instance of the operation and performing the input parameter association. The last element of the refinement sequence of an invocation is the termination action which associates the output parameters and deletes the operation instance.

Concurrency between user activities is modeled by the interleaved execution of sequences of actions belonging to different action refinements of operations being called by the users' representatives.

An object is a protected entity and its methods determine the access rights which can be granted to users. The set of rights is given with $R$.

For each object $k \in \mathcal{K}$ of the system a special state object called access control list is introduced. The access control list is used to protect objects from unauthorized access. The value of an access control list $a c l_{k}$ in a given state $s$ of the system is given by $s\left[a c l_{k}\right]$. The value of $a c l_{k}$ may change dynamically according to changing access restrictions concerning object $k$.

At a given time a user may perform different tasks within the system and each task may require special access rights. We introduce the notion of a role to abstractly describe the task of a user (for instance the administrator role or the statistic role for users performing statistical analyses). The set of roles is given with $R L$ and the mapping $r l_{b}$ associates with each user a set of roles. For each role of a user there must exist a user representative acting in behalf of the user in the specific role. This relationship is expressed by

\footnotetext{
${ }^{2} R$ may include an owner-right (c.f. [BL75]).
} 
the mapping $r l_{k}$. The access control list of an object describes, for example, the allowed access for users or for roles.

The object security model enables to model fine-grained protected entities (the objects of set $\mathcal{K}$ ) adapted to the level of granularity the system being modeled requires. The operations of the set $\mathcal{O}$ define well-formed transactions, i.e. access rights for well-formed operations can be modeled. The role mechanism enables to grant a user different sets of access rights depending on his specific task within the system.

\section{Example 1:}

We will model a simple message transport system with the object security model, but neglecting the action parts. The system consists of an object buffer which buffers messages and three user representatives sending and receiving messages in behalf of two users $U_{1}$ and $U_{2}$. send and receive are the methods of the object buffer. Messages are stored in the state object queue.

Security requirements: Permission to access the operation send should be granted to users performing tasks in the role SENDER and to the user $U_{1}$. Permission to access the operation receive should only be granted to users working with the role RECEIVER, and the user may not invoke the method receive.

A system enforcing these requirements can be modeled as follows:

The set of users is given by $\mathcal{B}=\left\{U_{1}, U_{2}\right\}$, and $R L=\{S E N D E R, R E C E I V E R\}$ defines the set of roles.

The set of user representatives is given by $\mathcal{K}^{\prime}=\left\{\right.$ sender $_{1}$, sender $_{2}$, receiver $\}$. buffer $\in \mathcal{K}$ is a passive object with the associated access control list acl $_{b u f f e r}$.

The mappings are defined by:

uـrep $\left(U_{1}\right)=\left\{\right.$ sender $_{1}$, receiver $\}$, u $\operatorname{rep}\left(U_{2}\right)=\left\{\right.$ sender $\left._{2}\right\}$;

$r l_{b}\left(U_{1}\right)=\{S E N D E R, R E C E I V E R\}, r l_{b}\left(U_{2}\right)=\{S E N D E R\}$;

$r l_{k}\left(\right.$ sender $\left._{1}\right)=\{S E N D E R\}, r l_{k}\left(\right.$ sender $\left._{2}\right)=\{S E N D E R\}$,

$r l_{k}($ receiver $)=\{R E C E I V E R\}$.

In the initial state $\left\{s_{0}\right\}=S_{0}$ the following property holds for the access control list $a_{b u f f e r}$ of the object buffer :

$$
\begin{aligned}
& (S E N D E R,\{\text { send }\}) \in s_{0}\left[\text { acl }_{b u f f e r}\right] \wedge\left(U_{1},\{\text { send }\}\right) \in s_{0}\left[\text { acl }_{\text {buffer }}\right] \\
& \wedge(R E C E I V E R,\{\text { receive }\}) \in s_{0}\left[\text { acl buffer }_{\text {bu }}\right]
\end{aligned}
$$

$\Delta$

\subsubsection{Influences and Observations between Users}

The influence and observation properties of actions will be re-defined in the context of the object security model, i.e. we will define information flows between users.

Definition 7 Influences between users:

Given a system $\mathcal{M S}$, the finite prefix $<A>$ of an accepted sequence $<A^{\prime}>\in \mathcal{A}^{\Delta}$, 
$s_{0} \stackrel{<A>}{\longrightarrow} s_{r}$ with $s_{0} \in S_{0}$ and the associated computation $\sigma_{A}$, two user representatives $u r_{1}, u r_{2} \in \mathcal{K}^{\prime}$, two operations $o p_{1}, o p_{2} \in \mathcal{O}$, a state object $v \in V$, and a state $s_{l} \in \sigma_{A}$.

User representative $u r_{1}$ influences user representative $u r_{2}$ in the sequence $s_{l}, \ldots, s_{r}$ of $\sigma_{A}$ by means of the state object $v$ and operations $o p_{1}$ and $o p_{2}$ if and only if the predicate in $f_{k}$ is true in state $s_{r}$.

$\omega\left(i n f_{k}\left(l, u r_{1}, u r_{2}, o p_{1}, o p_{2}, v\right), s_{r}\right)=$ true $: \Longleftrightarrow \exists i n s t \_o p_{1} \quad \exists i n s t \_o p_{2}:$

inst_op $p_{1}$ is an invocation of $o p_{1}$ invoked by $u r_{1}$ in $\sigma_{A}$ and

inst_op $p_{2}$ is an invocation of $o p_{2}$ invoked by $u r_{2}$ in $\sigma_{A}$ and

$\exists a \in \rho\left(\right.$ inst_op $\left.p_{1}\right) \exists b \in \rho($ inst_op $): \omega\left(i n f_{a}(l, a, b, v), s_{r}\right)=$ true

We say, there exists an influence between user representatives $u r_{1}$ and $u r_{2}$ with respect to operations $o p_{1}$ and $o p_{2}$ in a given computation if the action refinement of an invocation inst_op $p_{1}$ of operation $o p_{1}$ invoked by $u r_{1}$ contains an action which influences an action contained in the action refinement of an invocation inst_op $p_{2}$ of operation $o p_{2}$ invoked by $u r_{2}$. As user representatives act in behalf of users an information flow between those users is established.

The influence property defined in 7 is a generalization of the non-interference property of Goguen and Meseguer [GM82], because our model describes concurrent systems as well. Moreover, based on the action model the internal actions of the systems and their effects can be modeled and, therefore, the properties of these actions with respect to influences and observations can be determined. This allows us in contrast to [GM82] to model systems which create classified information caused by the execution of such internal actions.

\section{Example 2:}

Consider again the message transport system given in example 1 . Let $<A 1>$ be a sequence of actions which contains the execution of the actions of the user representatives sender $_{1}$ and sender $_{2}$ to enqueue new messages in the state object queue, as well as, the actions of the user representative receiver to dequeue a message from queue. The actions of the sender ${ }_{1}$ have been executed prior to the actions of the sender ${ }_{2}$ and of the receiver. The following predicates hold for the computation $\sigma_{A 1}, s_{0} \stackrel{<A 1\rangle}{\longrightarrow} s_{r}$ :

$$
\begin{gathered}
\omega\left(\text { in }_{k}\left(0, \text { sender }_{1}, \text { receiver, send, receive, queue }\right), s_{r}\right)=\text { true }, \\
\omega\left(\text { inf }_{k}\left(0, \text { sender }_{1}, \text { sender }_{2}, \text { send, send, queue }\right), s_{r}\right)=\text { true } .
\end{gathered}
$$

That is, the execution of an invocation of operation send invoked by sender $_{1}$ influences the execution of invocations receive and send invoked by receiver and sender $r_{2}$, respectively.

To be able to distinguish between information flows which can be encapsulated and hidden within an operation and those which are observable outside of an operation we translate the property of observability to the object security model. 
Definition 8 Observations between users:

Given the assumptions of definition 7.

User representative $u r_{2}$ observes user representative $u r_{1}$ in the sequence $s_{l}, \ldots, s_{r}$ of $\sigma_{A}$ by means of the state object $v$ and operations $o p_{2}$ and $o p_{1}$ if and only if the predicate $o b s_{k}$ is true in state $s_{r}$.

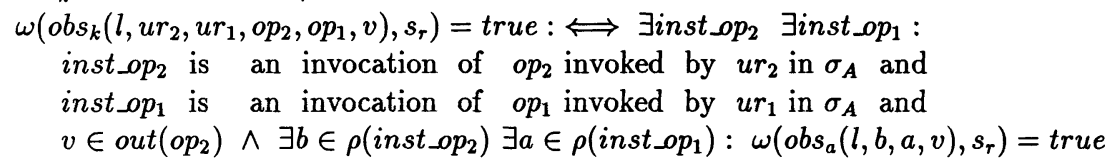

The activity of a user representative $u r_{1}$ is observable by a representative $u r_{2}$ if the value of an output parameter of at least one operation instance executed by $u r_{2}$ depends on the effects of the execution of at least one operation instance executed by $u r_{1}$.

\section{Example 3:}

Given the sequence of actions $\langle A 1>$ as described in example 2. Let $y$ be the output parameter of operation receive and let out $($ send $)=\emptyset$.

Security requirements (cont.): In addition to the security requirements given in example 1 concerning the access restrictions of the system we require that there may not exist an observable information flow between the two senders of the system.

The following two predicates hold:

$$
\text { (1) } \omega\left(\text { obs }_{k}\left(0, \text { receiver, sender } r_{1}, \text { receive, send }, y\right), s_{r}\right)=\text { true }
$$

(2) $\forall v \in V: \omega\left(\right.$ obs $_{k}\left(0\right.$, sender $_{2}$, sender $_{1}$, send, send, $\left.\left.v\right), s_{r}\right)=$ false

Predicate (1) is true in computation $\sigma_{A 1}$, because the execution of the send operation is observable by executing the receive operation.

Predicate (2) is false, because the send operation does not specify any output parameters, and, therefore, no observable influences may occur. That is, the influence of sender 1 on sender ${ }_{2}$ according to the influence property in $f_{k}$ stated in example 2 is encapsulated within operation send and is therefore not observable by user $U_{2}$ associated with the user representative sender ${ }_{2}$. User $U_{2}$ may not observe any activity of user $U_{1}$ by executing the operation send in computation $\sigma_{A 1}$. This non-observability property holds for each computation and the system enforces the security requirement stated above.

\section{$\Delta$}

The definitions introduced describe the semantics of influences and observations between users of a system in a universal way. We are free to chose an appropriate specification and implementation language to design secure systems. We just have to define an action semantics for the selected languages.

The security requirements of a system - the security policy - must be stated in terms of the allowed or disallowed influences and observations between the subjects of the system, as well as, in terms of the permitted accesses to system objects.

In the next section we will introduce the logic formulas used to specify security policies. 
The semantics of the formulas are formalized based on the influence and observe properties of the underlying models.

Within our framework systems and seeurity policies are uniformly modeled and specified. The uniform formalism enables the verification of security properties with respect to specified requirements. Furthermore, within the universal framework it is possible to compare and evaluate security policies.

\section{Security Requirements}

To specify disallowed influences and observations between users we will define conditional non-influencing requirements and conditional non-observing requirements. Disallowed and allowed accesses are specified by access restriction requirements. Definition 9 introduces the logic to specify security requirements.

Definition 9 Security requirement logic:

Given an object security model $\mathcal{M S}$. The set of state objects $V$ of $\mathcal{M S}$, a set of predicate symbols $P S$ with $\{c n i n f, c n o b s, a c c, i n\} \subseteq P S$, and the temporal operator $\square$ form the syntactic basis of the logic.

For each computation $\sigma$ of $\mathcal{M S}$ let < define the linear ordering on states of the sequence $\sigma$. Let $T F O$ be the set of all formulas over the syntactic basis. $\omega: T F O \times \sigma \longrightarrow\{$ true, false $\}$ defines the semantics of formulas in a computation $\sigma$ of $\mathcal{M S}$. Given Cond $\in T F O$, $u r_{1}, u r_{2} \in \mathcal{K}^{\prime}, o p_{1}, o p_{2} \in \mathcal{O}$, and $k \in \mathcal{K}$ the mapping $\omega$ is defined as follows:

1. Given a formula $P \in T F O$ :

$\omega(\square P, s)=$ true $: \Longleftrightarrow \forall s^{\prime} \in \sigma: s^{\prime} \leq s: \omega\left(P, s^{\prime}\right)=$ true.

2. The semantics of the conditional non-influencing predicate cninf is defined by:

$\omega\left(\operatorname{cninf}\left(u r_{1}, u r_{2}, o p_{1}, o p_{2}, C o n d\right), s\right)=$ true $: \Longleftrightarrow$

$\omega((\omega($ Cond,$s)=$ true $\Longrightarrow P), s)=$ true with $\mathrm{P}$ is given by

$P: \exists s^{\prime} \in \sigma: \forall v \in V: \omega\left(\right.$ inf $\left._{k}\left(s^{\prime}, u r_{1}, u r_{2}, o p_{1}, o p_{2}, v\right), s\right)=$ false with

$s^{\prime}<s \wedge \omega\left(\right.$ Cond, $\left.s^{\prime}\right)=$ true $\wedge \forall s^{\prime \prime} \in \sigma: s^{\prime}<s^{\prime \prime}<s$ holds $: \omega\left(\right.$ Cond, $\left.s^{\prime \prime}\right)=$ true $\wedge$

$s^{\prime} \notin S_{0} \Longrightarrow\left(\exists s^{\prime \prime \prime} \in \sigma: s^{\prime \prime \prime} \longrightarrow s^{\prime} \wedge \omega\left(\right.\right.$ Cond,$\left.s^{\prime \prime \prime}\right)=$ false $)$.

3. The semantics of the conditional non-observing predicate cnobs is defined by:

$\omega\left(\right.$ cnobs $\left(u r_{2}, u r_{1}, o p_{2}, o p_{1}\right.$, Cond $\left.), s\right)=$ true $: \Longleftrightarrow$

$\omega((\omega($ Cond,$s)=$ true $\Longrightarrow Q), s)=$ true with $\mathrm{Q}$ is given by

$Q: \exists s^{\prime} \in \sigma: \forall v \in V: \omega\left(o b s_{k}\left(s^{\prime}, u r_{2}, u r_{1}, o p_{2}, o p_{1}, v\right), s\right)=$ false with

$s^{\prime}$ is the state in the state sequence $\sigma$ with the same properties as stated above.

4. The semantics of the access restriction predicate acc is defined by:

$\omega\left(\operatorname{acc}\left(u r_{1}, o p, k\right.\right.$, Cond $\left.), s\right)=$ true $: \Longleftrightarrow$

$\omega((\omega($ Cond,$s)=$ false $\Longrightarrow R), s)=$ true with $\mathrm{R}$ is given by

$R: \nexists a \in e n(s): a$ is the first action of an action refinement sequence

of an invocation of op invoked by $u r_{1}$. 
5. $\omega($ in $(u r, o p), s)=$ true $: \Longleftrightarrow$ there exists an invocation inst_op of operation op invoked by representative $u r$ and the last element of the action refinement sequence $\rho($ inst_op $)$ has not been executed in the prefix $\sigma^{\prime}=s_{0}, \ldots, s$ of $\sigma$.

The temporal operator $\square$ expresses the usual 'henceforth' semantics.

A conditional non-influencing requirement between two user representatives $u r_{1}$ and $u r_{2}$ with respect to two operations requires that as long as the condition Cond within the requirement specification holds there may not occur an influence from $u r_{1}$ on $u r_{2}$ by executing the specified operations. The meaning of a conditional non-observing requirement can be stated in an analogues way. An access restriction requirement concerning a user representative, an object and an operation exported by the object requires that the representative may not execute the specified operation if the condition Cond within the requirement does not hold.

With the predicate in we are able to express control conditions. This is useful if we want to restrict, for instance, granted access rights to be used only within well-defined execution domains (see example below).

The conditional predicates offer a powerful formalism to specify security requirements tailored to the individual requirements of applications. With the access restriction predicates access control policies may be specified. Dynamically changing access rights may be expressed by specifying appropriate conditions within the predicates, for example, with conditions concerning the value of the access control list of the object being protected. Fine-grained information flow restrictions may be specified by using the cninf and cnobs predicates.

\section{Example 4:}

1. Given the ordered set of labels $L=\{$ unclassified, confidential, secret, top secret $\}$ and the labeling function $l: \mathcal{K}^{\prime} \longrightarrow L$.

The formula:

$$
\forall o p, o p^{\prime} \in \mathcal{O} \square c n i n f\left(u r_{1}, u r_{2}, o p, o p^{\prime},\left(l\left(u r_{1}\right)>l\left(u r_{2}\right)\right)\right.
$$

requires that there may not exist an influence from $u r_{1}$ on $u r_{2}$ if the classification of $u r_{1}$ is greater than the classification of $u r_{2}$. If we specify such a requirement for all user representatives and for all operations we will obtain the requirements of the multi-level security policy of the Bell LaPadula model [BL75].

2. Replacing the non-influencing formula given above with the non-observing formula:

$$
\forall o p, o p^{\prime} \in \mathcal{O} \square \operatorname{cnobs}\left(u r_{2}, u r_{1}, o p, o p^{\prime},\left(l\left(u r_{2}\right)<l\left(u r_{1}\right)\right)\right.
$$

weakens the multi-level security requirements by just disallowing observable information flows from higher to lower levels. This specification still covers the intended information flow requirements but is less restrictive than the original multi-level security policy. 
3. The formula:

$$
\square a c c\left(u r, o p, k,\left(a s s \_u(u r),\{o p\}\right) \in a c l_{k} \wedge \text { in }\left(u r, o p^{\prime}\right)\right)
$$

specifies that user representative $u r$ is permitted to access operation op of $k$ if its associated user $a s s \_u(u r)$ is contained in the access control list of $k$ and if the access to $o p$ is performed within the execution of operation $o p^{\prime}, i n\left(u r, o p^{\prime}\right)$. That is, the right to access $o p$ is restricted to a specified execution context.

\section{$\Delta$}

The examples demonstrate the expressiveness of the logic formulas. Requirements to restrict an allowed access to certain execution domains or to restrict the access by other conditions can not be expressed by the formalism of access control matrix approaches. The non-observability requirement of the example shows that multi-level security requirements can be specified in a less restrictive way, i.e. requirements are specified which are not expressible by the known formalisms.

We are now able to specify security policies for a system.

\subsection{Security Policies}

Definition 10 Security policy:

Given a system $\mathcal{M S}$. A security policy $P$ for $\mathcal{M S}$ is a formula of the security requirement logic defined by a conjunction of one initial formula, of non-influencing, non-observing, and of access restriction formulas.

An initial formula $P_{0}$ specifies the allowed and disallowed accesses in the initial states $s_{0} \in S_{0}$.

The security of a system is a measure of the system's ability to enforce a security policy $P$. A computation of the system is called secure with respect to $P$, if formula $P$ holds in each state of the computation. The system $\mathcal{S}$ is called secure with respect to $P$ if the formula $P$ holds in each computation of $\mathcal{S}$.

\section{Example 5:}

A security policy $P_{M T S}$ of the simple message transport system (MTS) may now be stated formally:

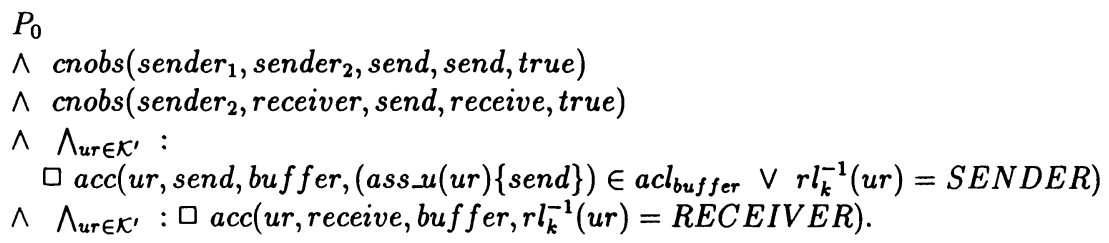


with

$$
\begin{gathered}
P_{0}=(S E N D E R,\{\text { send }\}) \in s_{0}\left[\text { acl }_{\text {buffer }}\right] \wedge\left(U_{1},\{\text { send }\}\right) \in s_{0}\left[\text { acl }_{\text {buffer }}\right] \\
\wedge(R E C E I V E R,\{\text { receive }\}) \in s_{0}[\text { acl buffer }]
\end{gathered}
$$

$P_{0}$ is the initial formula of $P_{M T S}$. The access restrictions permit the accesses to object buffer according to the informally stated restrictions in example 1 . The policy specifies that user $\mathrm{U} 2$ is not allowed to observe the activities of user $U_{1}$. In example 3 we have already argued that this non-observability property holds for each computation of the system. The system is secure with respect to policy $P_{M T S}$.

\section{Example 6:}

We will specify the Brewer and Nash [BN89] Chinese Wall security policy with the formalism of the security requirement logic. According to the Chinese Wall model the objects of the system are grouped together in company data sets containing objects concerning the same company and in conflict of interest classes containing company data sets whose companies are in competition. The labeling functions $x$ and $y$ specify for each object the conflict of interest class and the company data set, respectively. Public available information is labeled with the special company data set $y_{0}$. We introduce the special state object $N$ to store the access history of the system. $N$ is a boolean matrix with $N(k, u r)=$ true if user representative $u r$ has accessed object $k$.

Now we are prepared to specify the policy.

In the initial states of the system all users are permitted to access any object, i.e. the initial formula is given with $P_{0}=$ true.

For each passive object $k \in \mathcal{K}$ and for all operations $o p \in E(k)$ we specify access restriction formulas:

$$
\bigwedge_{u r \in \mathcal{K}^{\prime}}: \square \operatorname{acc}\left(u r, o p, k, \forall k^{\prime} \in \mathcal{K}:\left(N\left(k^{\prime}, u r\right)=\text { true } \Longrightarrow x\left(k^{\prime}\right) \neq x(k) \vee y\left(k^{\prime}\right)=y(k)\right)\right) .
$$

That is, access is only permitted for $u r$ if $k$ is in the same data set as an object $k^{\prime}$ already accessed by $u r$ or if $k$ belongs to a completely different conflict of interest class.

The information flow restrictions of the Chinese Wall policy are specified by conditional non-influencing formulas for each user representative ur and for each pair of passive objects $k$ and $k^{\prime}$ :

$$
\square \operatorname{cninf}\left(u r, u r, k . r e a d, k^{\prime} . w r i t e, y(k) \neq y\left(k^{\prime}\right) \wedge y(k) \neq y_{0}\right)
$$

That is, write access to object $k^{\prime}$ (denoted by $\mathrm{k}^{\prime}$.write) is not permitted if there does exist any object $k$ which has already been read by $u r$ and which is a member of a different company data set and which does not contain public informations.

With the specified formulas the Chinese Wall policy is given.

\section{$\Delta$}

Given a system modeled by the object security model. Different policies may be stated for the system. The formal basis presented permits to define a partial dominance ordering on these policies. We say, a policy $\mathrm{P} 1$ dominates a policy $\mathrm{P} 2$ if $\mathrm{P} 1$ is at least as restrictive as P2, i.e. information flows which are not allowed according to policy P2 are not allowed 
according to P1, too, and each disallowed access in policy P2 is disallowed in P1 as well. Consider for instance example 4. Replacing the cninf requirements with cnobs requirements now permits information flows which are not allowed with respect to the cninf specification. The policy consisting of the $\operatorname{cninf}$ formulas dominates the policy consisting of the cnobs formulas.

The formalism provides an appropriate basis for comparing security requirements and thus for strengthening or weakening the requirements systematically by adding or removing information flow or access restrictions or by strengthening or weakening the conditions within the requirement formulas.

\section{Conclusion}

We have presented a uniform framework to model secure concurrent systems and to specify security requirements. The action model provides the sound basis needed to formalize security properties of the modeled system. Based on an action model we introduced our object security model. With the formalism of the object security model fine-grained protected entities, together with access rights for well-formed operations, and application specific user roles defining protection domains for users can easily be modeled. The object security model models concurrent systems with integrity and confidentiality properties. Security requirements for secure distributed systems should be formally stated to enable the verification of the system's security properties with respect to specified requirements. The security requirement logic presented offers a powerful formalism to express fine-grained information flow restrictions as well as access restrictions tailored to the individual needs of applications. We have demonstrated the expressiveness of the logic by specifying well-known security policies, and requirements which can not be expressed by the known formalisms.

We are able to specify access control and information flow policies uniformly by using the formulas of the logic. This uniform specification framework offers for the first time the possibility to formally compare and evaluate security policies.

The models together with the security requirement logic can be applied to each level of implementation during the software engineering process of developing systems. On each level an action semantics of the language (for instance specification language, or highlevel programming language) to be used to implement the system must be defined and the formalism of the framework is usable to specify and verify security properties on the selected level of abstraction.

To implement specified security policies we have extended a high-level programming language with language concepts for access and information control. The development and implementation of appropriate operating system services based on a Mach3.0-Kernel to systematically realize secure systems in a physically distributed environment is still ongoing. 


\section{References}

[BL75] D.E. Bell and L. LaPadula. Secure Computer Systems: Unified Exposition and MULTICS Interpretation. Technical Report MTR - 2997, MITRE Corp, Bedford, July 1975 .

[BN89] D.F.C. Brewer and M.J. Nash. The Chinese Wall Security Policy. In Proceedings 1989 IEEE Symposium on Security and Privacy, pages 206 - 214, 1989.

[CW87] D.D. Clark and D.R. Wilson. A Comparison of Commercial and Military Computer Security Policies. In Proceedings of the 1987 IEEE Symposium on Security and Privacy, pages 184 - 194, 1987.

[GM82] J.A. Goguen and J. Meseguer. Security Policies and Security Models. In Proceedings of the 1982 IEEE Symposium on Security and Privacy, pages 11 - 20, 1982. 